\title{
On the essential spectrum of the many-particle Schrödinger operator with combined Zeeman and Stark effect
}

\author{
By \\ Masaharu ARAI*
}

\section{Introduction}

Let us consider a system consisting of $N_{o}$ infinitely heavy nuclei and $N$ electrons which is exposed to an external electromagnetic field, and thus whose Schrödinger operator $H$ is given by

$$
\begin{aligned}
H u(x) & =\sum_{j=1}^{N} \sum_{\nu=0}^{2}\left(i \frac{\partial}{\partial x_{3 j-\nu}}+b_{\nu}(x)\right)^{2} u(x)- \\
& -\sum_{j=1}^{N} \sum_{i=1}^{N_{0}} \frac{A_{i}}{\left|r_{j}-a_{i}\right|} u(x)+ \\
& +\sum_{1 \leqq j<k \leqq N} \frac{1}{\left|r_{j}-r_{k}\right|} u(x)-\sum_{j=1}^{N} B x_{3 j} u(x),
\end{aligned}
$$

where $r_{j}=\left(x_{3 j-2}, x_{3 j-1}, x_{3 j}\right)$ and $a_{i}=\left(a_{3 i-2}, a_{3 i-1}, a_{3 i}\right)$ are the position vectors of the $j$-th electron and of the $i$-th nucleus, respectively, and $A_{i}$ and $B$ are positive constants, $b_{\nu}(x)$ are real functions of class $C^{1}$.

$H$ is formally selfadjoint, and moreover, it determines a unique selfadjoint operator in Hilbert space $\mathfrak{Q}=L_{2}\left(K^{3 N}\right)$; see [3].

Žislin [9], Jörgens [4] anc' others have investigated the spectrum of $H$ when the external electric field (the last term in (1.1)) does not exist. But as for the spectrum of $H$ with the external electric field, no complete study seems to have been made yet except a work of Titchmarsh [8] (p. 134). He considered a special case of (1.1) with $N_{0}=N=1$, and $b_{\nu}(x)=0$, that is,

Received September 7, 1967.

Communicated by S. Matsuura.

* Department of Mathematics, Nihon University. 


$$
H u(x)=-\Delta u(x)-\frac{A}{|x|} u(x)-B x_{3} u(x),
$$

where $x=\left(x_{1}, x_{2}, x_{3}\right) \in R^{3}$, and $A$ and $B$ are positive constants. He showed that the spectrum of $H$ defined by (1.2) is continuous on the entire real axis.

In this paper we shall study the spectrum of $H,(1.1)$, with arbitrary $N_{0}$ and $N$, and show that the essential spectrum of $H$ is the entire real axis. It is still unknown whether $H$ has a point spectrum or not.

\section{Statement of the result}

Let us consider the operator of the form

$$
T u(x)=\sum_{j=1}^{m}\left(i \frac{\partial}{\partial x_{i}}+b_{j}(x)\right)^{2} u(x)+q(x) u(x)
$$

in the whole space $R^{m}(m \geqq 3)$. Denote by $T$ the most general operator that is significant whenever the right-hand side of (2.1) is defined in the distribution sense.

Before writing down our assumptions on $T$, we shall give some definitions.

Definition 1. For a fixed constant $\alpha>0$, let us denote by $Q_{\alpha, l o c}$ the set of all functions $p(x)$ for each of which

$$
M_{p}(x)=\int_{|x-y| \leqq 1} \frac{|p(y)|^{2}}{|x-y|^{m^{-4+\alpha}}} d y
$$

is locally bounded. Let us denote by $Q_{\alpha}$ the set of all $p \in Q_{\alpha, l o c}$ such that $M_{p}(x)$ is uniformly bounded over $R^{m}$.

Definition 2. Let $\alpha_{i}, \beta_{i}(j=1,2, \cdots, m)$ be constants such that $0<\alpha_{j}<\beta_{j}$, and $\gamma$ is a positive constant. Let

$$
D=\left\{x=\left(x_{1}, x_{2}, \cdots, x_{m}\right) ; \alpha_{j} t \leqq x_{j} \leqq \beta_{j} t, j=1,2, \cdots, m, t \geqq \gamma\right\} .
$$

Let us call a domain $D$ of this type a "cone".

We assume:

(A) $b_{j}(x)$ are real valued functions of class $C^{1}$. 
(B) $q(x)=q^{(1)}(x)+q^{(2)}(x)$, where $q^{(1)} \in Q_{\alpha, \text { loc }}$ and $q^{(2)} \in Q_{\alpha}$ such that there exists a positive non-decreasing function $q^{*}(r)$ of $r \geqq 0$ such that $q^{(1)}(x) \geqq-q^{*}(|x|)$ and $\int^{\infty} q^{*}(r)^{-1 / 2} d r=+\infty$.

(C) There exist a "cone" $D$ with $\alpha_{j}, \beta_{j}$ and $\gamma$ as in Definition 2, and a constant $\varepsilon, 0<\varepsilon<2$, and non-empty subset $J$ of the indices $\{1,2, \cdots, m\}$, such that the following conditions are fulfilled:

$\left(\mathrm{C}_{1}\right)$ In $D, q^{(1)}(x)$ is reduced to the sum of one-dimensional functions $q_{j}\left(x_{j}\right), j=1,2, \cdots, m$, of class $C^{2}$ defined on $x_{j} \geqq \alpha_{j} \gamma$, that is,

$$
q^{(1)}(x)=\sum_{j=1}^{m} q_{j}\left(x_{j}\right) \quad x=\left(x_{1}, x_{2}, \cdots, x_{m}\right) \in D
$$

such that each $q_{j}\left(x_{j}\right)$ with $j \in J$ is non-increasing tending to $-\infty$ as $x_{j} \rightarrow+\infty$, and satisfies

Const. $\geqq q_{j}\left(x_{j}\right) \geqq-$ Const. $x_{j}^{2-\varepsilon}$ 1)

and

$$
\left.\int^{\infty}\left|\frac{q_{j}^{\prime \prime}(t)}{\left|q_{j}(t)\right|^{3 / 2}}-\frac{5}{4} \frac{q_{j}^{\prime}(t)^{2}}{\left|q_{j}(t)\right|^{5 / 2}}\right| d t<+\infty, 2\right)
$$

and such that each $q_{j}\left(x_{j}\right)$ with $j \notin J$, if it exists, converges to a finite value $\mu_{j}$ as $x_{j}$ tends to $+\infty$;

$\left(\mathrm{C}_{2}\right)$ In $D, q^{(2)}(x)$ is continuous and converges to zero as $|x| \rightarrow+\infty$;

$\left(\mathrm{C}_{3}\right)$ In $D$, there exist constants $b_{j}^{0}$ such that

$$
b_{j}(x)-b_{j}^{0}=o\left(|x|^{-1+\varepsilon / 2}\right) \text { as }|x| \rightarrow+\infty,
$$

and furthermore

$$
\sum_{j=1}^{m} \frac{\partial b_{j}(x)}{\partial x_{j}} \rightarrow 0 \text { as }|x| \rightarrow+\infty
$$

The list of our assumptions is over.

Let $A$ be an arbitrary differential operator interpreted in the distribution sense. Lec $A_{0}$ be the restriction of $A$ with domain $C_{0}^{\infty}\left(R^{m}\right)$ (the set of all functions of class $C^{\infty}$ on $R^{m}$ with compact support).

1) Here and henceforth Const. signifies any positive constant. not necessarily the same.

2) The similar condition to (2.5) is imposed by N. Dunford and J.T. Schwartz to study the essential spectrum of Strum-Liouville problem; see [1] chap. XIII §. 6.22. 
As is well known, if $A_{0}$ is essentially selfadjoint, its unique selfadjoint extension, its closure and its adjoint $A_{0}^{*}$ coincide with each other. Henceforth we shall use $A_{0}^{*}$ to denote any of them.

Let only (A) and (B) be satisfied. By (B), $q \in L_{2, \text { loc }}$, and thus $T_{0}$ is a symmetric operator in $\mathfrak{Q}=L_{2}\left(R^{m}\right)$. Here we recall a theorem of Ikebe and Kato [3].

Theorem 1. (Ikebe-Kato) ${ }^{3)}$ Let $(A)$ and $(B)$ be satisfied. Then $T_{0}$ is essentially selfadjoint, and its unique selfadjoint extension $T_{0}^{*}$ is the restriction of $T$ with domain

$$
\mathscr{D}\left(T_{0}^{*}\right)=\left\{u \in L_{2} ; u \in H_{2, l o c},{ }^{4)} \quad T u \in L_{2}\right\} .
$$

Our aim is to show

Theorem 2. Under the assumptions $(A),(B)$ and $(C)$, the essential spectrum of $T_{0}^{*}$ is the entive real axis.

Let us return to the operator $H$ defined in (1.1). We can apply Theorem 2 to this operator, and have the following result.

Theorem 3. If $b_{\nu}(x)$ are real valued functions of class $C^{1}$ and there exist real constants $b_{\nu}^{0}$ such that

$$
b_{\nu}(x)-b_{\nu}^{0}=o\left(|x|^{-1 / 2}\right) \text { as }|x| \rightarrow+\infty,
$$

and

$$
\sum_{\nu=0}^{2} \frac{\partial b_{\nu}(x)}{\partial x_{3 j-\nu}} \rightarrow 0 \text { as }|x| \rightarrow+\infty \text {, for any } j,
$$

then the essential spectrum of $H_{0}^{*}$ is the entire real axis.

Proof. Put

$$
\begin{aligned}
& q^{(1)}(x)=-\sum_{j=1}^{N} B x_{3 j}, \\
& q^{(2)}(x)=-\sum_{j=1}^{N} \sum_{\imath=1}^{N_{0}} \frac{A_{i}}{\left|\gamma_{j}-a_{i}\right|}+\sum_{j<k} \frac{1}{\left|r_{j}-r_{k}\right|},
\end{aligned}
$$

and let us show that conditions (A), (B) and (C) are satisfied. (A)

3) See [3], Theorem 3 and Lemma 4.

4) $H_{2,1 \text { oc }}$ is the set of all locally $H_{2}$-functions, where $H_{2}$ is the completion of $C_{0}^{\infty}$ with the second-order Dirichlet norm

$$
\|u\|_{2}=\left\{\Sigma_{j k}, \|\left.\frac{\partial^{2}}{\partial x_{j} \partial x_{k}} u\right|_{L_{2}\left(R^{m}\right)} ^{2}+\left.\Sigma_{j}|| \frac{\partial}{\partial x_{j}} u\right|_{L_{2}\left(R^{m}\right)} ^{\prime 2}+\left|u_{\mid}^{\prime}\right|_{L_{2}\left(R^{m}\right)}^{2}\right\}^{1 / 2} .
$$


is assumed here. $q^{(1)}(x) \in Q_{\alpha, l o c}$ with $q^{*}(r)=N \cdot B \cdot r$ which satisfies $q^{(1)}(x) \geqq-q^{*}(|x|)$ and $\int^{\infty} q^{*}(r)^{-1 / 2} d r=+\infty$. As to the interaction potential $q^{(2)}(x)$, Stummel [7] (p. 163) has shown that $q^{(2)}(x) \in Q_{\alpha}$. Thus (B) is satisfied, and by Theorem $1 H_{0}$ has a unique selfadjoint extension $H_{0}^{*}$.

Put $J=\{3 j ; j=1,2, \cdots, N\}$, and $\varepsilon=1$. Then for any choice of a "cone" $D,\left(C_{1}\right)$ and $\left(C_{3}\right)$ can be seen to be satisfied. It is easy to see that there exists a "cone" $D$ satisfying $\left(C_{2}\right)$. Thus (C) is satisfied, and Theorem 2 yields the result.

Remark. Let (A) and (B) are satisfied. Since assumption (C) depends upon the choice of the Cartesian coordinate axes, but $(A)$ and (B) do not, we note that under the change of Cartesian coordinate axes, $T$ remairs of type (2.1), and thus if (C) is satisfied under a suitable choice of the Cartesian coordinate axes, then the conclusion of Theorem 2 is true.

\section{One dimensional problem}

Let $A$ be a selfadjoint operator acting in an abstract Hilbert space with the norm \|\| . We introduce $\sigma(A)$ to denote the spectrum of $A$, and $\sigma_{e}(A)$, its essential spectrum.

The next lemma will be of frequent use.

Lemma 1.5) $\lambda \in \sigma_{e}(A)$ if and only if there exists a sequence $\left\{u_{n}\right\}_{n=0}^{\infty}$ in $\mathscr{D}(A)$ sucn that

(a) $\left\|u_{n}\right\|=1$,

(b) $\left\{u_{n}\right\}$ has no strongly convergent subsequence,

(c) $A u_{n}-\lambda u_{n} \rightarrow 0$ strongly as $n \rightarrow+\infty$.

Let us call this sequence $\left\{u_{n}\right\}$ a singular sequence of $A$ corresponding to $\lambda$.

If $q^{(2)}(x)=0, b_{j}(x)=0$ and the decomposition (2.3) is true in the whole space $R^{m}$, then our operator becomes

$$
-\Delta u(x)+\sum_{j=1}^{m} q_{j}\left(x_{j}\right) u(x),
$$

5) See [1]. XIII, $\$ 7.12$. 
and this operator is separable. Therefore in this section we shall investigate ordinary differential operators.

Let

$$
L u(t)=-\frac{d^{2}}{d t^{2}} u(t)+q(t) u(t)
$$

in $a \leqq t \leqq+\infty$, where $-\infty<a<+\infty$ and $q(t)$ is a real valued continuous function. Let $L_{a}$ be the restriction of $L$ with domain $C_{0}^{2}(a$, + $\infty$ ) (the set of all functions of class $C^{2}$ on $(a,+\infty)$ with compact support). Since $L_{a}$ is a real symmetric operator in $\mathfrak{F}_{2}=L_{2}[a,+\infty)$, it has selfadjoint extensions. Let us denote one of them by $\widetilde{L_{a}}$.

In the remainder of this section let $\mathfrak{2}=L_{2}[a,+\infty)$ and denote by ( , ) and \|\| the ordinary inner product and norm in $\mathfrak{g}$.

The following lemmata are known.

Lemma 2. ${ }^{6)}$ All the selfadjoint extensions of $L_{a}$ have the same essential spectrum.

Lemma 3. ${ }^{7} \partial_{e}\left(\widetilde{L_{a}}\right)$ does not depend upon the choice of $a$, as far as $q \in C[a,+\infty)$.

By the last two lemmata, we can write $\sigma_{e}(L)$ instead of $\sigma_{e}\left(\widetilde{L}_{a}\right)$. The last lemma shows that when the essential spectrum of $\widetilde{L}_{a}$ is concerned, the left end point $a$ of the interval on which the operator is considered can be chosen as large as necessary. Henceforth, $a$ is taken as large as necessary without notice.

Lemma $4 .^{8)}$ Let $K_{n}=[n \alpha, n \beta], K_{n}^{\prime}=\left[n \alpha^{\prime}, n \beta^{\prime}\right]$, for $0<\alpha<\alpha^{\prime}<$ $\beta^{\prime}<\beta$. If the equation $L f=\lambda f$ in $t \geqq a$ has a non-trivial solution of class $C^{2}$ satisfying that

6) See [6] Kap. V §19 Satz 2 (s. 203).

7) See Ibid. $\$ 19$ Bemerkung 2 (s. 200), or see [5] Theorem 22.

8) This lemma is a modification of the following theorem proved by Hartman and Wintner [2]. But our proof is different from theirs, for we want to gain a singular sequence with the properties (3.1) and (3.2).

Theorem: If the equation $L f=\lambda f$ has a solution $f(t) \risingdotseq 0$, satisfying

$$
\int_{a}^{\iota}\left|f^{\prime}(s)\right|^{2} d s=0\left(t^{2}\right)
$$

then either $f(t)$ is of class $L_{2}$ or $\lambda$ is in the essential spectrum of $L$. 
(F.1) $f(t)$ is bounded i.e. $|f(t)| \leqq M$,

(F. 2) $M_{1}(n) \equiv \int_{K_{n}^{\prime}}|f(t)|^{2} d t \rightarrow+\infty$ as $n \rightarrow+\infty$,

(F. 3) $M_{2}(n) \equiv \int_{K_{n}}\left|f^{\prime}(t)\right|^{2} d t=O\left(n^{2}\right)$ as $\rightarrow+\infty$,

then there exists a singular sequence $\left\{u_{n}(t)\right\}$ corresponding to $\lambda$, whose terms are as smooth as $f$, and have the following properties:

The supports of $u_{n}(t)$ are contained in $K_{n}$ for large $n$, $\int\left|u_{n}^{\prime}(t)\right|^{2} d t \leqq$ Const. $\left(1+M_{2}(n)\right) \cdot M_{1}(n)^{-1}$.

In particular $\lambda \in \sigma_{\theta}(L)$.

Proof. Let $\rho(t)$ and $\rho_{n}(t)$ be $C_{0}^{\infty}$ functions with the following properties: $0 \leqq \rho(t) \leqq 1, \rho(t)=0$ if $t \leqq \alpha$ or $t \geqq \beta, \rho(t)=1$ if $\alpha^{\prime} \leqq t \leqq \beta^{\prime}$ : $\rho_{n}(t)=\rho(t / n)$. Let $n$ be as large as $n \alpha \geqq a$. Put $f_{n}(t)=f(t) \rho_{n}(t)$, then

$$
\left\|f_{n}\right\|^{2}=\int|f|^{2} \rho_{n}^{2} d t \geqq \int_{K_{n}^{\prime}}|f|^{2} d t=M_{1}(n) \rightarrow+\infty .
$$

We put $u_{n}(t)=f_{n}(t) /\left\|f_{n}\right\|$, which evidently satisfies conditions (a), (b) of Lemma 1, and (3.1). Let us show that $\left\{u_{n}(t)\right\}$ satisfies (c) of Lemma 1.

$$
\begin{aligned}
& f_{n}^{\prime \prime}=\left(f \cdot \rho_{n}\right){ }^{\prime \prime}=f^{\prime \prime} \cdot \rho_{n}+2 f^{\prime} \cdot \rho_{n}^{\prime}+f \cdot \rho_{n}^{\prime \prime}, \\
& L f_{n}-\lambda f_{n}=(L f-\lambda f) \cdot \rho_{n}-2 f^{\prime} \cdot \rho_{n}^{\prime}-f \cdot \rho_{n}^{\prime \prime} \\
& =-2 f^{\prime} \cdot \rho_{n}^{\prime}-f \cdot \rho_{n}^{\prime \prime} .
\end{aligned}
$$

Since

$$
\left|\rho_{n}^{\prime}(t)\right| \leqq \text { Const. } n^{-1}, \quad\left|\rho_{n}^{\prime \prime}\right| \leqq \text { Const. } n^{-2},
$$

it follows from (F.3) and (3.3) that

$$
\frac{\left\|f^{\prime} \cdot \rho_{n}^{\prime}\right\|^{2}}{\left\|f_{n}\right\|^{2}} \leqq \frac{\text { Const. }}{n^{2}} \int_{K_{n}}\left|f^{\prime}\right|^{2} d t \cdot M_{1}(n)^{-1} \rightarrow 0 ;
$$

and from (F.1), (F.2) and (3.3) that

$$
\begin{aligned}
\frac{\left\|f \cdot \rho_{n}^{\prime \prime}\right\|_{1}^{2}}{\left\|f_{n}\right\|^{2}} & \leqq \frac{\text { Const. }}{n^{4}} \int_{K_{n}}|f|^{2} d t \cdot M_{1}(n)^{-1} \\
& \leqq \frac{\text { Const. } M^{2}(\beta-\alpha) n}{n^{4}} M_{1}(n)^{-1} \rightarrow 0
\end{aligned}
$$


as $n \rightarrow+\infty$. Thus we have

$$
L u_{u}-\lambda u_{u}=-\frac{2 f^{\prime} \cdot \rho_{n}^{\prime}}{\left\|f_{n}\right\|}-\frac{f \cdot \rho_{n}^{\prime \prime}}{\left\|f_{n}\right\|} \rightarrow 0 \text { as } n \rightarrow+\infty .
$$

and hence

$$
\lambda \in \sigma_{e}(L) .
$$

Now we shall show property (3.2).

$$
\begin{aligned}
& u_{n}^{\prime}(t)=\left(\rho_{n}^{\prime}(t) f(t)+\rho_{n}(t) f^{\prime}(t)\right) /\left\|f_{n}\right\|, \\
& \left|u_{n}^{\prime}(t)\right| \leqq\left(\text { Const. } / n+\left|f^{\prime}(t)\right|\right) /\left\|f_{n}\right\|,
\end{aligned}
$$

and thus

$$
\begin{aligned}
& \int\left|u_{n}^{\prime}(t)\right|^{2} d t=\int_{K_{n}}\left|u_{n}^{\prime}(t)\right|^{2} d t \leqq \frac{\text { Const. }}{n^{2}} \frac{(\beta-\alpha) n}{\left\|f_{n}\right\|^{2}}+ \\
+ & \frac{\text { Const. } \int_{K_{n}}\left|f^{\prime}\right|^{2} d t}{\left\|f_{n}\right\|^{2}} \leqq \text { Const. }\left(1+M_{2}(n)\right) \cdot M_{1}(n)^{-1} \quad \text { q. e. d. }
\end{aligned}
$$

Lemma 5. Let $q(t)$ be continuous and tend to a finite value $\mu$ as $t \rightarrow+\infty$, and let $K_{n}=[n \alpha, n \beta]$ for constants $\alpha$ and $\beta, 0<\alpha<\beta$. Then $\sigma_{\epsilon}(L)=[\mu,+\infty)$, and for any $\lambda \in \sigma_{e}(L)$ there exists a singular sequence $\left\{u_{n}(t)\right\}$ of class $C_{0}^{\infty}$ with properties (3.1) and

$$
\int\left|u_{n}^{\prime}(t)\right|^{2} d t \leqq \text { Const. }
$$

Proof. For any $c<\mu$, there exists $a$, such that for all $t \geqq a$, $q(t) \geqq c$. As the spectrum of an operator is contained in the closure of its numerical range,

$$
\sigma_{e}(L)=\sigma_{e}\left(\tilde{L}_{a}\right) \subset \sigma\left(\tilde{L}_{a}\right) \subset \text { closure of }\left\{\lambda=\left(\tilde{L}_{a} u, u\right) ; u \in \mathscr{D}\left(\tilde{L}_{a}\right),\|u\|=1\right\} \text {. }
$$

Since

$$
\left(\tilde{L}_{a} u, u\right)=-\left(u^{\prime \prime}, u\right)+(q u, u) \geqq c\|u\|^{2}=c \text { for }\|u\|=1,
$$

we have $\sigma_{e}(L) \subset[c,+\infty)$ for any $c<\mu$, which implies

$$
\sigma_{e}(L) \subset[\mu,+\infty) \text {. }
$$

Next let us show the reverse inclusion. We put

$$
L^{\prime}=-\frac{d^{2}}{d t^{2}}+\mu
$$

Then $L=L^{\prime}+\tilde{q}(t)$, where $\tilde{q}(t) \equiv q(t)-\mu$ tends to zero as $t$ tends to 
infinity. Let $K_{n}^{\prime}=\left[n \alpha^{\prime}, n \beta^{\prime}\right]$ with $0<\alpha<\alpha^{\prime}<\beta^{\prime}<\beta$. For any $\lambda \geqq \mu$, the equation $L^{\prime} f=\lambda f$ has a solution $f(t)=\sin (\sqrt{\lambda-\mu} t)$, which satisfies the conditions of Lemma 4 . Hence $\lambda \in \sigma_{e}\left(L^{\prime}\right)$.

Let $f_{n}(t)$ and $u_{n}(t)$ be functions constructed in the same way as in the proof of Lemma 4 with the solution $f(t)=\sin (\sqrt{\lambda-\mu} t)$ of $L^{\prime} f=\lambda f$. Then $\left\{u_{n}(t)\right\}$ has properties (a), (b) of Lemma 1 , and (3.1). By (3.1),

$$
\tilde{q} u_{n} \rightarrow 0 \text { strongly in } L_{2} \text {, }
$$

and thus

$$
L u_{n}-\lambda u_{n}=L^{\prime} u_{n}-\lambda u_{n}+\tilde{q} u_{n} \rightarrow 0 \text { strongly in } L_{2} .
$$

This shows that $\lambda$, and $\left\{u_{n}\right\}$ are also a point of the essential spectrum and a singular sequence of $L$, respectively. Thus $\sigma_{e}(L)=[\mu,+\infty)$.

Since

$$
\left\|f_{n}\right\|^{2} \geqq \int_{K n}|f|^{2} d t \geqq \text { const. } n
$$

it follows from (3.4) that

$$
\left|u_{n}^{\prime}(t)\right|^{2} \leqq\left(\text { Const. } / n+\left|f^{\prime}(t)\right|\right)^{2} /\left\|f_{n}\right\|^{2} \leqq \text { Const. } n^{-1} .
$$

Integrating both side over $K_{n}$, we have (3.5).

q. e. d.

Lemma 6. Let $q(t)$ be of class $C^{2}$ and be non-increasing tending to $-\infty$, and statisfy

$$
\text { Const. } \geqq q(t) \geqq- \text { Const. } t^{2-\varepsilon} \quad(0<\varepsilon<2)
$$

and

$$
\int^{\infty}\left|\frac{q^{\prime \prime}(t)}{|q(t)|^{3 / 2}}-\frac{5}{4} \frac{q^{\prime}(t)^{2}}{|q(t)|^{5 / 2}}\right| d t<+\infty
$$

Let $K_{n}=[n \alpha, n \beta]$ with $0<\alpha<\beta$. Then $\sigma_{e}(L)=(-\infty,+\infty)$, and for any real $\lambda$ there exists a singular sequence of class $C_{0}^{2}$ with properties:

(3.1) The supports of $u_{n}(t)$ are contained in $K_{n}$ for large $n$, and

$$
\left\|u_{n}^{\prime}\right\|^{2}=O\left(n^{2-\varepsilon}\right) \text { as } n \rightarrow+\infty \text {. }
$$

Proof. For any real $\lambda, p(t) \equiv \lambda-q(t)$ is a positive function for 
sufficiently large $t$, say $t \geqq a$. And (4.7) is also true for $q(t)$ replaced by $p(t) . \quad L f=\lambda f$ becomes

$$
f^{\prime \prime}(t)+p(t) f(t)=0
$$

It is known $n^{9)}$ that if $p(t)$ is positive and satisfies (3. 7), for $q(t)$ replaced by $p(t)$ then the above equation (3.9) has a solution $f(t)$ such that

$$
\left\{\begin{array}{l}
f(t)=p(t)^{-1 / 4}(\sin s(t))(1+o(1)) \\
f^{\prime}(t)=p(t)^{1 / 4}(\cos s(t))(1+o(1))
\end{array}\right.
$$

as $t \rightarrow \infty$, where

$$
s(t)=\int_{a}^{t} p(t)^{1 / 2} d t, \quad s^{\prime}(t)=p(t)^{1 / 2},
$$

Let $K_{n}^{\prime} \equiv\left[n \alpha^{\prime}, n \beta^{\prime}\right] \quad$ with $\quad 0<\alpha<\alpha^{\prime}<\beta^{\prime}<\beta$.

Let us show that this solution $f(t)$ satisfies assumptions (F. 1), (F. 2) and (F. 3) of Lemma 4. If this is shown, we can see by the arbitrariness of $\lambda$, that $\sigma_{e}(L)=(-\infty,+\infty)$, and that there exists a singular sequence with property (3.1). Let $n$ be as large as $n \alpha \geqq a$. As $p(t)$ is non-decreasing (F.1) is obviously satisfied. As $p(t)=O\left(t^{2-\varepsilon}\right)$,

$$
\left.M_{2}(n) \equiv \int_{K_{n}} f^{\prime}(t)\right|^{2} d t \leqq 2 \int_{K_{n}} p(t)^{1 / 2} d t=O\left(n^{2-\varepsilon / 2}\right),
$$

and thus (F. 3) holds.

In order to show (F.2), it is sufficient to show the inequality

$$
M_{1}(n) \equiv \int_{K_{n}^{\prime}}|f|^{2} d t \geqq \text { Const. } n^{\varepsilon / 2} \quad \text { for large } n \text {. }
$$

put

$$
I_{n} \equiv \int_{K^{\prime} n} p(t)^{-1 / 2} \sin ^{2} s(t) d t
$$

and

$$
J_{n} \equiv \int_{K^{\prime} n} p(t)^{-1 / 2} d t
$$

9) Cf. [1], XIII, $\$ \$ 6.18-6.20$, where only the first line of (3.10) is given. The second one can be seen by calculating the behavior of the derivative in each step of the proof loc. cit. 
Using the triangle inequality in $L_{2}\left(K_{n}^{\prime}\right)$,

$$
\begin{gathered}
M_{1}(n)^{1 / 2}=\left\{\int_{K_{n}^{\prime}}|f(t)|^{2} d t\right\}^{1 / 2} \geqq\left\{\int_{K_{n}^{\prime}} p(t)^{-1 / 2} \sin ^{2} s(t) d t\right\}^{1 / 2}- \\
-o(1)\left\{\int_{K^{\prime} n} p(t)^{-1 / 2} \sin ^{2} s(t) d t\right\}^{1 / 2} \geqq \frac{1}{2} \cdot I_{n}^{1 / 2}, \quad \text { for large } n .
\end{gathered}
$$

Changing the variable,

$$
\begin{aligned}
I_{n} & =\int_{\alpha_{n}}^{\beta_{n}} \sin ^{2} s \cdot p(t(s))^{-1 / 2} \cdot p(t(s))^{-1 / 2} d s \\
& =\int_{\alpha_{n}}^{\beta_{n}} \sin ^{2} s \cdot p(t(s))^{-1} d s,
\end{aligned}
$$

where the function $t(s)$ is defined by $t(s(t))=t$, and $\alpha_{n}$ and $\beta_{n}$ are defined by

$$
\alpha_{n}=s\left(n \alpha^{\prime}\right)=\int_{a}^{n \alpha^{\prime}} p(t)^{1 / 2} d t, \beta_{n}=s\left(n \beta^{\prime}\right)=\int_{a}^{n \beta^{\prime}} p(t)^{1 / 2} d t
$$

Since

$$
\beta_{n}-\alpha_{n}=\int_{n \alpha^{\prime}}^{n \beta \prime} p(t)^{1 / 2} d t \rightarrow+\infty \text { as } n \rightarrow+\infty,
$$

we can assume that $\beta_{n}-\alpha_{n} \geqq \pi / 2$. Since $p(t)$ and $t(s)$ are non-decreasing,

$$
\begin{aligned}
& \int_{\alpha_{n}+\pi / 2}^{\beta_{n}} \cos ^{2} s \cdot p(t(s))^{-1} d s=\int_{\alpha_{n}+\pi / 2}^{\beta_{n}} \sin ^{2}(s-\pi / 2) \cdot p(t(s))^{-1} d s \\
\leqq & \int_{\alpha_{n}+\pi \mathbb{s}}^{\beta_{n}} \sin ^{2}(s-\pi / 2) \cdot p(t(s-\pi / 2))^{-1} d s \\
= & \int_{\alpha_{n}}^{\beta_{n}-\pi / 2} \sin ^{2} s \cdot p(t(s))^{-1} d s \leqq \int_{\alpha_{n}}^{\beta_{n}} \sin ^{2} s \cdot p(t(s))^{-1} d s=I_{n} .
\end{aligned}
$$

Considering the fact that

$$
\int_{\alpha_{n}}^{\alpha_{n}+\pi / 2} p(t(s))^{-1} d s \leqq \text { Const. }
$$

we have

$$
J_{n}=\int_{K_{n}^{\prime}} p(t)^{-1 / 2} d t=\int_{\alpha_{n}}^{\beta^{*}}\left(\sin ^{2} s+\cos ^{2} s\right) p(t(s))^{-1} d s
$$


$\leqq$ Const. $+2 I_{n}$.

On the other hand

$$
\begin{aligned}
J_{n} & =\int_{K n} \underset{K^{\prime}}{p}(t)^{-12} d t \geqq \text { Const. } \int_{n \alpha^{\prime}}^{n \beta \prime} t_{2}^{-1+\varepsilon / 2} d t \\
& =\text { Const. } n^{\varepsilon / 2} .
\end{aligned}
$$

By (3. 14) and (3.15) we have

$$
I_{n} \geqq\left(J_{n}-\text { Const. }\right) / 2 \geqq \text { Const. } n^{\varepsilon / 2} \text {, }
$$

for large $n$. By this and (3.13) we have

$$
M_{1}(n)^{1 / 2} \geqq \text { Const. } n^{\varepsilon / 4} \text {, }
$$

and thus (3.12) holds.

Let $f_{n}(t)$ and $u_{n}(t)$ be functions constructed in the same way as in the proof of Lemma 4 with the above solution $f(t)$ and given $K_{n}$ and $K_{n}^{\prime}$.

Then from (3.2), (3.11) and (3.12)

$$
\left\|u_{n}^{\prime}\right\|^{2}=\left\{1+O\left(n^{2-\varepsilon / 2}\right)\right\} \cdot O\left(n^{-\varepsilon / 2}\right)=O\left(n^{2-\varepsilon}\right) .
$$

Thus (3.8) holds and $\left\{u_{n}(t)\right\}$ is a desired sequence.

q. e. $d$ 。

It should be noted that in Lemmata 5 and 6 the choice of $\alpha$ and $\beta$ which define the family of intervals $K_{n}$ are arbitrary, if only $0<\alpha<\beta$.

\section{Proof of Theorem 2}

Let

$$
\begin{aligned}
& K_{n}^{(j)}=\left\{t ; n \alpha_{j} \leqq t \leqq n \beta_{j}\right\}, j=1,2, \ldots, m, \\
& K_{n}=K_{n}^{(1)} \times K_{n}^{(2)} \times \cdots \times K_{n}^{(m)},
\end{aligned}
$$

where $\alpha_{j}$ and $\beta_{j}$ are the numbers which define the "cone" $D$ in assumption (C). We give the proof in several steps.

1st step. Suppose $b_{j}(x)=0$ and $q^{(2)}(x)=0$. Then our operator becomes

$$
S u=-\Delta u(x)+q^{(1)}(x) u(x) .
$$

In this step we shall show that $\sigma_{e}\left(S_{0}^{*}\right)=(-\infty,+\infty)$ and for any $\lambda$ 
there exists a singular sequence $\left\{u_{n}(x)\right\}$ with the properties: The supports of $u_{n}(x)$ are contained in $K_{n}$. $\int\left|\operatorname{grad} u_{n}(x)\right|^{2} d x=O\left(n^{2-\varepsilon}\right), \quad$ as $n \rightarrow+\infty$,

where $\varepsilon$ is the constant in condition (C).

For any real $\lambda$, let us choose $\lambda_{j}, j=1,2, \ldots, m$, such that $\lambda_{j} \geqq \mu_{j}$ if $j \notin J$ and such that the equation $\lambda=\sum_{j=1}^{m} \lambda_{j}$ is satisfied.

If $j \notin J$, then by Lemma 5 , and if $j \in J$, then by Lemma $6, \lambda_{j}$ is a point of the essential spectrum of the operator

$$
L_{j} u(t)=-\frac{d^{2}}{d t^{2}} u(t)+q_{j}(t) u(t) .
$$

Let us denote by $\left\{u_{n}^{(j)}(t)\right\}$ the singular sequence of $L_{j}$ corresponding to $\lambda_{j}$, constructed in the same way as in the proof of Lemma 5 or 6 , where $K_{n}^{(j)}$ can be taken as in (4.1) by what was noted at the end of the previous section. Put

$$
u_{n}(x)=u_{n}^{(1)}\left(x_{1}\right) \cdot u_{n}^{(2)}\left(x_{2}\right) \cdots u_{n}^{(m)}\left(x_{m}\right) .
$$

Then $u_{n}(x)$ has property (4.3). Since $u_{n}^{(j)}\left(x_{j}\right) \in C_{0}^{2}, u_{n}(x) \in C_{0}^{2}\left(R^{m}\right)$, and so by virtue of Theorem $1 u_{n}(x) \in \mathscr{D}\left(S_{0}^{*}\right)$.

Let us show that $\left\{u_{n}(x)\right\}$ is a singular sequence corresponding to $\lambda$ of the operator $S_{0}^{*}$, that is, it has properties (a), (b) and (c) of lemma 1. Properties (a) and (b) hold obviously. To prove (c) we consider

$$
\begin{aligned}
& S_{0}^{*} u_{n}-\lambda u_{n}=-\Delta u_{n}(x)+\sum_{j=1}^{n} q_{j}\left(x_{j}\right) u_{n}(x)-\lambda u_{n}(x) \\
= & -\sum_{j=1}^{n} \frac{d^{2}}{d x_{j}^{2}} u_{n}^{(j)}\left(x_{j}\right) \cdot \prod_{k \neq j} u_{n}^{(k)}\left(x_{k}\right)+\sum_{j=1}^{m} q_{j}\left(x_{j}\right) \cdot \prod_{k=1}^{m} u_{n}^{(k)}\left(x_{k}\right)- \\
& \quad-\lambda \prod_{k=1}^{m} u_{n}^{(k)}\left(x_{k}\right) \\
= & \sum_{j=1}^{n n}\left(L_{j} u_{n}^{(j)}\left(x_{j}\right)-\lambda u_{n}^{(j)}\left(x_{j}\right)\right) \cdot \prod_{k \neq j} u_{n}^{(k)}\left(x_{k}\right),
\end{aligned}
$$

where we used (4.3) and that $q^{(1)}(x)=\sum_{j=1}^{m} q_{j}\left(x_{j}\right)$ in $x \in \bigcup_{n} K_{n} \subset D$. Since

$$
\left\{\begin{array}{l}
\left\|u_{n}^{(j)}\right\|_{L_{2}\left(R^{1}\right)}=1 \text { for } j=1,2, \ldots, m ; n=1,2, \ldots, \\
\left\|L_{j} u_{n}^{(j)}-\lambda u_{n}^{(j)}\right\|_{L_{2}\left(R^{1}\right)} \rightarrow 0 \text { as } n \rightarrow+\infty, \text { for } j=1,2, \ldots, m,
\end{array}\right.
$$

we have 


$$
\left\|S_{0}^{*} u_{n}-\lambda u_{n}\right\|_{L_{2}\left(R^{m}\right)} \rightarrow 0 \text { as } n \rightarrow+\infty,
$$

and thus $(C)$ holds and $\left\{u_{n}\right\}$ is a singular sequence.

Next let us show property (4.4). Since

$$
\begin{aligned}
& \left|\operatorname{grad} u_{n}(x)\right|^{2}=\sum_{j=1}^{n !}\left|\frac{\partial}{\partial x_{j}} u_{n}(x)\right|^{2} \\
& =\left.\sum_{j=1}^{m}\left|\frac{d}{d x_{j}} u_{n}^{(n)}\left(x_{j}\right)^{\mid 2} \cdot \prod_{k \neq j}\right| u_{n}^{(k)}\left(x_{k}\right)\right|^{2},
\end{aligned}
$$

considering (4.6), we have

$$
\begin{aligned}
& \left\|\operatorname{grad} u_{n}\right\|_{L_{2}\left(R^{m}\right)}^{2}=\int\left|\operatorname{grad} u_{n}(x)\right|^{2} d x \\
& =\sum_{j=1}^{m}\left\|\frac{d}{d x_{j}} u_{n}^{(j)}\right\|_{L_{2}\left(R^{1}\right)}^{2} \times \prod_{k \neq j}\left\|u_{n}^{(k)}\right\|_{L_{2}\left(R^{1}\right)}^{2} \\
& =\sum_{j=1}^{m}\left\|\frac{d}{d x_{j}} u_{n}^{(j)}\right\|_{L_{2}\left(R^{1}\right)}^{2}
\end{aligned}
$$

Each $\left\|\frac{d}{d x_{j}} u_{n}^{(j)}\right\|_{L_{2}(R 1)}^{2}$ is estimated by (3.5) if $j \notin J$, and by (3.8) if $j \in J$, and thus (4.4) holds.

2nd step. Assume that all $b_{j}^{0}$ are zero, then our operator becomes

$$
T^{\prime}=S+2 i \sum_{j=1}^{m} b_{j}(x) \frac{\partial}{\partial x_{j}}+i \sum_{j=1}^{m} \frac{\partial b_{j}(x)}{\partial x_{j}}+\sum_{j=1}^{m} b_{j}(x)^{2}+q^{(2)}(x) .
$$

Let us show that the singular sequence $\left\{u_{n}(x)\right\}$ of $S_{0}^{*}$ constructed in the lst step is also a singular sequence of $T_{0}^{\prime *}$ corresponding to the same $\lambda$. As $u_{n}(x) \in \mathscr{D}\left(T_{0}^{\prime *}\right)$ follows from Theorem 1 and the fact that $u_{n}(x) \in C_{0}^{2}$, it is sufficient to show that

$$
\begin{aligned}
& \left\|b_{j}(x) \frac{\partial u_{n}}{\partial x_{j}}\right\|_{L_{2}\left(R^{m}\right)} \rightarrow 0, \\
& \|\left.\sum_{j=1}^{m} \frac{\partial b_{j}(x)}{\partial x_{j}} u_{n}(x)\right|_{L_{2}\left(R^{m}\right)} \rightarrow 0 . \\
& \left\|b_{j}(x)^{2} u_{n}(x)\right\|_{L_{2}\left(R_{u l}\right)} \rightarrow 0, \text { for } j=1,2, \ldots, m, \\
& \left\|q^{(2)}(x) u_{n}(x)\right\|_{L_{2}\left(R^{m}\right)} \rightarrow 0,
\end{aligned}
$$

as $n \rightarrow+\infty$.

Assertion (4.7) follows from (2.6), (4.3) and (4.4). In fact, as- 
sumption (2.6) with $b_{j}^{0}=0$ shows that for any $\delta>0$, there exists a real number $R_{\delta}$ such that for any $x$ in $D \cup\left\{|x|>R_{s}\right\}$

$$
\frac{\left|b_{j}(x)\right|}{|x|^{-1+\varepsilon / 2}}<\delta
$$

By (4.3), the supports of $b_{j}(x) \frac{\partial u_{n}}{\partial x_{j}}$ are contained in $K_{n} \subset D \cap$ $\{x ;|x| \geqq n \alpha\}$, where $\alpha=\min _{1 \leqq j \leqq m} \alpha_{j}$. Fix a number $N_{\delta}$ such that $\alpha N_{\delta} \geqq R_{\delta}$. For any $n>N_{\delta}$, from (4.11) we have

$$
\frac{\left|b_{j}(x)\right|}{(n \alpha)^{-1+\varepsilon / 2}}<\delta \text { for } x \in K_{n} .
$$

Considering (4.4), we have

$$
\begin{aligned}
& \left|b_{j}(x) \frac{\partial u_{n}}{\partial x_{j}}\right|_{L_{2}\left(R^{m}\right)}^{2}=\int_{K_{n}}\left|b_{j}(x)\right|^{2} \cdot \mid \frac{\partial u_{n}}{\partial_{j} x} d x \leqq \\
& \leqq \delta^{2} n^{-2+\varepsilon} \alpha^{-2+\varepsilon} \int\left|\frac{\partial u_{n}}{\partial x_{j}}\right|^{2} d x \leqq \text { Const. } \delta^{2}
\end{aligned}
$$

for any $n>N_{\delta}$. As $\delta$ is arbitrary, this shows that (4.7) holds.

Assertion (4.8) follows from (2.7) and (4.3). In fact, for any $\delta>0$, by (2.7), there exists a real number $R_{\delta}$ such that

$$
\left|\sum_{j=1}^{m} \frac{\partial b_{j}(x)}{\partial x_{j}}\right|<\delta \quad \text { in } \quad x \in D \cup\left\{|x| \geqq R_{\delta}\right\}
$$

For large $n$ such that $n \alpha \geqq R_{\delta}$, considering (4.3) we have

$$
\left\|\sum_{j=1}^{m} \frac{\partial b_{j}(x)}{\partial x_{j}} u_{n}(x)\right\|_{L_{2}\left(R^{m}\right)} \leqq \delta\left\|u_{n}\right\|_{L_{2}\left(R^{m}\right)}=\delta .
$$

Thus (4. 8) holds.

Finally, let us show (4.9) and (4.10) using (2.6) and $\left(C_{2}\right)$. From (2.6) with $b_{j}^{0}=0$ and $\varepsilon<2$, it follows that

$$
b_{j}(x) \rightarrow 0 \text { as }|x| \rightarrow+\infty \text { within } D .
$$

(4.12) and $\left(C_{2}\right)$ imply that for any $\delta>0$, there exists a real number $R_{\delta}$ such that

$$
\left|b_{j}(x)\right|<\delta \text { and }\left|q^{(2)}(x)\right|<\delta
$$

in $x \in D \cup\left\{|x| \geqq R_{\delta}\right\}$. For large $n$ such that $n \alpha \geqq R_{\delta}$, considering (4.3), 
we have

$$
\left\|b_{j}(x)^{2} \cdot u_{n}(x)\right\|_{L_{2}\left(R^{m}\right)} \leqq \delta^{2}\left\|u_{n}\right\|_{L_{2}\left(R^{m}\right)}=\delta^{2}
$$

and

$$
\left\|q^{(2)} u_{n}(x)\right\|_{L_{2}\left(R^{m}\right)} \leqq \delta\left\|u_{n}\right\|_{L_{2}\left(R^{m}\right)}=\delta .
$$

Thus (4.9) and (4.10) hold.

3rd step. Let us consider the general case. Put

$$
\begin{aligned}
& \vec{b}_{j}(x)=b_{j}(x)-b_{j}^{0}, \\
& b^{0} \cdot x=\sum_{j=1}^{m} b_{j}^{0} x_{j},
\end{aligned}
$$

and

$$
T^{\prime} u=\sum_{j=1}^{m}\left(i \frac{\partial}{\partial x_{j}}+b_{j}\right)^{2} u+q^{(1)} u+q^{(2)} u
$$

then $T^{\prime}$ satisfies assumptions in 2nd step, and thus $\sigma_{e}\left(T_{0}^{*}\right)=(-\infty,+\infty)$. Let $\lambda$ be an arbitrary real number and let $\left\{u_{n}\right\}$ be a singular sequence of $T^{\prime}$ corresponding to $\lambda$. Put

$$
v_{n}(x)=e^{i b^{0} \cdot x} u_{n}(x) \text {. }
$$

Since

$$
\begin{gathered}
\sum_{j=1}^{m}\left(i-\frac{\partial}{\partial x_{j}}+b_{j}(x)\right)^{2}\left(e^{i b^{0} \cdot x} u\right) \\
=e^{i b 0 \cdot x}\left[\sum_{j=1}^{m}\left(i \frac{\partial}{\partial x_{j}}+\vec{b}_{j}(x)\right)^{2} u,\right],
\end{gathered}
$$

we have

$$
T v_{n}-\lambda v_{n}=e^{i 0^{0} \cdot x}\left(T^{\prime} u_{n}-\lambda u_{n}\right) \rightarrow 0 \text { in } L_{2}\left(R^{m}\right),
$$

which shows that $\left\{v_{n}(x)\right\}$ is a singular sequence of $T_{0}^{*}$ corresponding to an arbitrarily given real $\lambda$. Therefore $\sigma_{t}\left(T_{0}^{*}\right)$ is $(-\infty,+\infty)$ and we complete the proof of Theorem 2 .

In conclusion, the writer wishes to express his sincere gratitude to Professor T. Ikebe for his enduring encouragements and kind instructions.

\section{REFERENCES}

[1] Dunford, N. and J. T. Schwartz, Linear operators, II, Interscience, New York, 1963. 
[2] Hartman, P. and A. Wintner, On the derivative of the solutions of one-dimensional wave equations, Amer. J. Math. 72 (1950), 148-155.

[3] Ikebe, T. an $\mathrm{I} T$. Kato, Uniqueness of the selfadjoint extentions of singular elliptic differential operators, Arch. Rational Mech. Anal. 9 (1962), 77-92.

[4] Jörgens, K., Über das wesentliche Spektrum elliptischer Differentialoperatoren vom Schrödinger-Typ, Universität Heidelberg, 1965.

[5] Kodaira, K., On eigenvalue problems associated with second-order differential equations, Sûgaku 2 (1949), 113-139. (Japanese)

[6] Neumark, M.A., Lineare Differentialoperatoren, Akademie Verlag, Berlin, 1960.

[7] Stummel, F., Singuläre elliptische Differentialoperatoren in Hilbertschen Räume, Math. Ann. 132 (1956), 150-176.

[8] Titchmarsh, E. C., Eigenfunction expantion associated with second-order differential equations, II, Oxford, 1958.

[9] Žislin, G, M., Discussion of the spectrum of the Schrödinger operator for the many-particle system, Trudy Mosk. Mat. Obšč. 9 (1960), 82-120. (Russian)

Note added in proof (October 25, 1967):

Recently, Professor M.S.P. Eastham studied some interesting "conditions for the spectrum in eigenfunction theory to consist of $(-\infty, \infty)$ ", Quart. J. Math. (2), 18 (1967), 147-153. One of them, that is, his Theorem 2 seems to be similar to our result. But when I want to apply his result to (1.1), I can only apply to the case reduced to (1.2). His Theorem 2 neither implies nor is implied by ours. 
